\title{
HOMOTHETIC MOTIONS IN THE GENERALIZED 3-SPACE
}

\section{Mehdi JAFARI ${ }^{1}$}

\begin{abstract}
A complete treatment of one parameter homothetic motions in three and four dimensional Euclidean spaces is provided in the Yayli's PhD thesis [15]. Here we follow his idea to define one parameter homothetic motion in generalized 3-space $\mathrm{E}_{\alpha \beta}^{3}$. By means of the generalized Hamilton operators, we also define a Hamilton motion and show that it is a homothetic motion. We investigate some properties of this motion and show that Darboux vector of the motion can be written as multiplication of two generalized quaternions.
\end{abstract}

Keywords: Darboux vector, generalize Hamilton operator, Generalized quaternion, Homothetic motion, Pole point.

\section{ÜÇ BOYUTLU GENELEŞTİRLMIŞ UZAYDA HOMOTETİK HAREKET}

\section{$\ddot{O} z$}

Üç ve dört boyutlu Öklid uzayinda, bir parametre homotetik hareketleri Yaylı'nın doktora tezin'de [15] tam olarak temın edilir. Burada biz bu fikri kulanarak, genelleştirilmiş 3-boyutlu uzayda bir parametre homotetik hareketi tanıtırdık. Genelleştirilmiş Hamilton operatörler ile, aynı zamanda Hamilton hareket tanımlar ve bu bir homototik hareket olduğunu göstermektedir.Bu hareketın bazı özelliklerini araştırdık ve gösterdik ki bu hareketin Darboux vektörü İki genelleştirilmiş kuaterniyonların çarpmasi yazilabilir.

Anahtar Kelimeler: Darboux vektör, Genelleştirilmiş Hamilton operatör, Genelleştirilmiş kuaterniyon, homotetik hareket, Pol noktası.

\section{INTRODUCTION}

In the Euclidean space $\mathrm{E}^{n}$, H.R. Müler [12] has studied the one-parameter singular motions and has given some characterizations for axoid surfaces. One-parameter homothetic motions of a rigid body in $n$-dimensional Euclidean space is investigated in [1] and some of its properties are given by Hacisalihoglu [2], showing that the motion is regular and has one pole point at every instant $t$. The homothetic motions in $E^{3}$ and $E^{4}$ via the Hamilton operators are studied by Yayl1 $[14,15]$. Subsequently, Kula and Yaylı [10] expressed the Hamilton motions by means of Hamilton operators in semi-Euclidean space $\mathrm{E}_{2}^{4}$ and have showed that these motions all are a homothetic motion. In Lorentz 4-space, properties of the homothetic motions are considered in [13]. In our previous work, a matrix corresponding to the Hamilton operators is defined for generalized quaternions, which determines a Hamilton motion in four-dimensional space $\mathrm{E}_{\alpha \beta}^{4}$. It is shown that this is a homothetic motion [6]. In this paper, the homothetic motions in an $n$-dimensional generalized space $\mathrm{E}_{\alpha \beta}^{3}$ are defined and some of their properties are investigated. Subsequently, with the aid of the generalized Hamilton operators, we define a Hamilton motion in three-dimensional space $\mathrm{E}_{\alpha \beta}^{3}$. We demonstrate that this motion is a one-parameter homothetic motion. We investigate some properties of this motion 
and show that Darboux vector of the motion can be written as multiplication of two generalized quaternions.

\section{Generalized Quaternions Algebra}

A generalized quaternion $q$ is an expression of the form

$$
q=a_{0}+a_{1} \vec{i}+a_{2} \vec{j}+a_{3} \vec{k}
$$

where $a_{0}, a_{1}, a_{2}$ and $a_{3}$ are real numbers and $\vec{i}, \vec{j}, \vec{k}$ are quaternionic units which satisfy the equalities

and

$$
\begin{aligned}
& \vec{i}^{2}=-\alpha, \quad \vec{j}^{2}=-\beta, \quad \vec{k}^{2}=-\alpha \beta, \\
& \vec{i} j=\overrightarrow{\mathrm{k}}=-\vec{j} \vec{i}, \quad \vec{j} \vec{k}=\beta \vec{i}=-\vec{k} \vec{j},
\end{aligned}
$$

$$
\overrightarrow{k i}=\alpha \vec{j}=-\overrightarrow{i k}, \quad \alpha, \beta \in \mathrm{R} .
$$

The set of all generalized quaternions are denoted by $\mathrm{H}_{\alpha \beta}$. A generalized quaternion $q$ is a sum of a scalar and a vector, where called scalar part is, $S_{q}=a_{0}$, and vector part is $\vec{V}_{q}=a_{1} \vec{i}+a_{2} \vec{j}+a_{3} \vec{k} \in \mathrm{E}_{\alpha \beta}^{3}$. If $S_{q}=0$, then $q$ is called pure generalized quaternion. The set of all the pure generalized quaternions is denoted by $\mathrm{K}$.

$\mathrm{H}_{\alpha \beta}$ is form a 4-dimensional real space which contains the real axis $\mathrm{R}$ and a 3-dimensional real linear space $\mathrm{E}_{\alpha \beta}^{3}$, so that, $\mathrm{H}_{\alpha \beta}=\mathrm{R} \oplus \mathrm{E}_{\alpha \beta}^{3}$.

\section{Special cases:}

1. $\alpha=\beta=1$, is considered, then $\mathrm{H}_{\alpha \beta}$ is the algebra of real quaternions.

2. $\alpha=1, \beta=-1$, is considered, then $\mathrm{H}_{\alpha \beta}$ is the algebra of split quaternions.

3. $\alpha=1, \beta=0$, is considered, then $\mathrm{H}_{\alpha \beta}$ is the algebra of semi-quaternions [11].

4. $\alpha=-1, \beta=0$, is considered, then $\mathrm{H}_{\alpha \beta}$ is the algebra of split semi-quaternions [8].

5. $\alpha=0, \beta=0$, is considered, then $\mathrm{H}_{\alpha \beta}$ is the algebra of quasi-quaternions[3].

If $q=\left(a_{0}, \vec{V}_{q}\right)$ and $p=\left(b_{0}, \vec{V}_{p}\right)$ are two quaternions, their sum is defined as

$$
q+p=\left(a_{0}+b_{0}, \vec{V}_{q}+\vec{V}_{p}\right)
$$

and their product (non-commutative) as

$$
q \circ p=\left(a_{0} b_{0}-\left\langle\overrightarrow{V_{q}}, \overrightarrow{V_{p}}\right\rangle, a_{0} \overrightarrow{V_{p}}+b_{0} \vec{V}_{q}+\vec{V}_{p} \times \vec{V}_{q}\right),
$$

here " $<,>$ " and " $\times$ " are the inner and vector products in $\mathrm{E}_{\alpha \beta}^{3}$, respectively. The conjugate quaternion of $q$ is defined as $\bar{q}=\left(a_{0},-\vec{V}_{q}\right)$ and the length or norm as

$$
N_{q}=q \circ \bar{q}=\bar{q} \circ q=a_{0}^{2}+\alpha a_{1}^{2}+\beta a_{2}^{2}+\alpha \beta a_{3}^{2} \in \mathrm{R} .
$$

Note that $N_{q p}=N_{q} N_{p}$. Every non-zero quaternion has a multiplicative inverse given by its conjugate divided by its norm: $q^{-1}=\bar{q} / N_{q}$. The generalized quaternion with a norm of one, $N_{q}=1$, is a unit generalized quaternion. 
If a generalized quaternion is looked at as a four-dimensional vector, the generalized quaternion product can be described by a matrix-vector product as

$$
q \circ p=\left[\begin{array}{cccc}
a_{0} & -\alpha a_{1} & -\beta a_{2} & -\alpha \beta a_{3} \\
a_{1} & a_{0} & -\beta a_{3} & \beta a_{2} \\
a_{2} & \alpha a_{3} & a_{0} & -\alpha a_{1} \\
a_{3} & -a_{2} & a_{1} & a_{0}
\end{array}\right]\left[\begin{array}{c}
b_{0} \\
b_{1} \\
b_{2} \\
b_{3}
\end{array}\right] .
$$

Let $q$ be a unit generalized quaternion, then $\stackrel{+}{h}_{q}: \mathrm{H}_{\alpha \beta} \rightarrow \mathrm{H}_{\alpha \beta}$ and $\bar{h}_{q}: \mathrm{H}_{\alpha \beta} \rightarrow \mathrm{H}_{\alpha \beta}$ are defined as follows:

$$
\stackrel{+}{h}_{q}(x)=q \circ x, \quad \bar{h}_{q}(x)=x \circ q \quad x \in \mathrm{H}_{\alpha \beta} .
$$

In both cases, considering $\mathrm{H}_{\alpha \beta}$ to be $\mathrm{E}_{\alpha \beta}^{4}$ spanned by the usual basic elements. We suspect that both these maps correspond to rotation, since it easy to show that they are norm and angle preserving. For example, considering the map $\stackrel{+}{h}$, we have already seen that if $x, y, q \in \mathrm{H}_{\alpha \beta}$ and $N_{q}=1$, then

$$
N_{q x}=N_{q} N_{x}=N_{x} \text {. }
$$

The generalized Hamilton operators $\stackrel{+}{H}$ and $\stackrel{-}{H}$, could be represented as the matrices;

$$
\stackrel{+}{H}(q)=\left[\begin{array}{cccc}
a_{0} & -\alpha a_{1} & -\beta a_{2} & -\alpha \beta a_{3} \\
a_{1} & a_{0} & -\beta a_{3} & \beta a_{2} \\
a_{2} & \alpha a_{3} & a_{0} & -\alpha a_{1} \\
a_{3} & -a_{2} & a_{1} & a_{0}
\end{array}\right]
$$

and

$$
\bar{H}(q)=\left[\begin{array}{cccc}
a_{0} & -\alpha a_{1} & -\beta a_{2} & -\alpha \beta a_{3} \\
a_{1} & a_{0} & \beta a_{3} & -\beta a_{2} \\
a_{2} & -\alpha a_{3} & a_{0} & \alpha a_{1} \\
a_{3} & a_{2} & -a_{1} & a_{0}
\end{array}\right] .
$$

A direct consequence of the above operators is the following identities:

$$
\stackrel{+}{H}(1)=I_{4}, \stackrel{+}{H}(\overrightarrow{\mathrm{i}})=E_{1}, \stackrel{+}{H}(\overrightarrow{\mathrm{j}})=E_{2}, \stackrel{+}{H}(\overrightarrow{\mathrm{k}})=E_{3},
$$

and

$$
\bar{H}(1)=I_{4}, \bar{H}(\overrightarrow{\mathrm{i}})=F_{1}, \bar{H}(\overrightarrow{\mathrm{j}})=F_{2}, \bar{H}(\overrightarrow{\mathrm{k}})=F_{3},
$$

where $I_{4}$ is a $4 \times 4$ identity matrix. Note, that the properties of the $E_{n}$ and $F_{n}(n=1,2,3)$ are identical to those of generalized quaternionic unit $\overrightarrow{\mathrm{i}}, \overrightarrow{\mathrm{j}}, \overrightarrow{\mathrm{k}}$. Since $\stackrel{+}{H}$ and $\vec{H}$ are linear, it follows that;

and

$$
\begin{aligned}
\stackrel{+}{H}(q) & =a_{0} \stackrel{+}{H}(1)+a_{1} \stackrel{+}{H}(\overrightarrow{\mathrm{i}})+a_{2} \stackrel{+}{H}(\overrightarrow{\mathrm{j}})+a_{3} \stackrel{+}{H}(\overrightarrow{\mathrm{k}}) \\
& =a_{0} I_{4}+a_{1} E_{1}+a_{2} E_{2}+a_{3} E_{3},
\end{aligned}
$$

$$
\begin{aligned}
\bar{H}(q) & =a_{0} \bar{H}(1)+a_{1} \bar{H}(\overrightarrow{\mathrm{i}})+a_{2} \bar{H}(\overrightarrow{\mathrm{j}})+a_{3} \bar{H}(\overrightarrow{\mathrm{k}}) \\
& =a_{0} I_{4}+a_{1} F_{1}+a_{2} F_{2}+a_{3} F_{3} .
\end{aligned}
$$

Using the definitions of $\stackrel{+}{H}$ and $\vec{H}$, the multiplication of the two generalized quaternions $q$ and $p$ is given by

$$
q \circ p=\stackrel{+}{H}(q) p=\stackrel{-}{H}(p) q .
$$


Theorem 1: If $q$ and $p$ are two generalized quaternions, $\lambda$ is a real number and $\stackrel{+}{H}$ and $\stackrel{-}{H}$ are operators as defined in equations (1) and (2), respectively, then the following identities hold:

$$
\begin{aligned}
& \text { i. } \quad q=p \Leftrightarrow \stackrel{+}{H}(q)=\stackrel{+}{H}(p) \Leftrightarrow \vec{H}(q)=\bar{H}(p) \text {. } \\
& \text { ii. } \stackrel{+}{H}(q+p)=\stackrel{+}{H}(q)+\stackrel{+}{H}(p), \quad \stackrel{-}{H}(q+p)=\vec{H}(q)+\bar{H}(p) \text {. } \\
& \text { iii. } \stackrel{+}{H}(\lambda q)=\lambda \stackrel{+}{H}(q), \stackrel{-}{H}(\lambda q)=\lambda \bar{H}(q) \text {. } \\
& \text { iv. } \quad \stackrel{+}{H}(q \circ p)=\stackrel{+}{H}(q) \stackrel{+}{H}(p), \quad \vec{H}(q \circ p)=\bar{H}(p) \vec{H}(q) \text {. } \\
& \text { v. } \quad \stackrel{+}{H}\left(q^{-1}\right)=[\stackrel{+}{H}(q)]^{-1}, \quad-\bar{H}\left(q^{-1}\right)=[-\vec{H}(q)]^{-1}, \quad\left(N_{q}\right)^{2} \neq 0 . \\
& \text { vi. } \quad \stackrel{+}{H}(\bar{q})=[\stackrel{+}{H}(q)]^{T}, \quad \bar{H}(\bar{q})=[-\vec{H}(q)]^{T} \text {. } \\
& \text { vii. } \operatorname{det}[\stackrel{+}{H}(q)]=\left(N_{q}\right)^{2}, \operatorname{det}[\dot{H}(q)]=\left(N_{q}\right)^{2} \text {. } \\
& \text { viii. } \operatorname{tr}[\stackrel{+}{H}(q)]=4 a_{0}, \operatorname{tr}[\dot{-}(q)]=4 a_{0} \text {. }
\end{aligned}
$$

Proof: The proof can be found in [4].

A matrix $A$ is called a quasi-orthogonal matrix if $A^{T} \varepsilon A=\varepsilon$ and $\operatorname{det} A=1$ where

$$
\varepsilon=\left[\begin{array}{ccc}
\alpha & 0 & 0 \\
0 & \beta & 0 \\
0 & 0 & \alpha \beta
\end{array}\right],
$$

and $\alpha, \beta \in \mathrm{R}$. The set of all quasi-orthogonal matrices, $\mathrm{QO}(3)$, with the operation of matrix multiplication is called rotations group in 3-spaces $\mathrm{E}_{\alpha \beta}^{3}[7]$.

\section{HOMOTHETIC MOTIONS AT $\mathrm{E}_{\alpha \beta}^{3}$}

In this section, we define one-parameter homothetic motion along a curve in a generalized 3space and show this motion satisfy all of the properties in Euclidean 3-space which is investigated by Yayl1 [15].

In three-dimensional generalized space $\mathrm{E}_{\alpha \beta}^{3}$, one-parameter homothetic motions of a body are generated by the transformation

$$
\left[\begin{array}{l}
Y \\
1
\end{array}\right]=\left[\begin{array}{cc}
h A & C \\
0 & 1
\end{array}\right]\left[\begin{array}{l}
X \\
1
\end{array}\right],
$$

where $A$ is a $3 \times 3$ quasi-orthogonal matrix and $h$ is homothetic scalar. The matrix $B=h A$ is called a homothetic matrix and $Y, X$ and $C$ are $3 \times 1$ real matrices. The homothetic scalar $h$ and the elements of $A$ and $C$ are continuously differentiable functions of a real parameter $t$.

To avoid the case of affine transformation we suppose that

$$
h(t) \neq \text { cons. }
$$

and to avoid the case of a pure translation or a pure rotation, we also suppose that

$$
\frac{d}{d t}(h A) \neq 0, \quad \frac{d}{d t}(C) \neq 0 .
$$


If we differentiate the equation $A^{T} \varepsilon A=\varepsilon$, we get

$$
\left(\dot{A}^{T} \varepsilon\right) A+A^{T} \varepsilon \dot{A}=0 .
$$

By choosing $A^{T} \varepsilon \dot{A}=\Omega$ and $\Omega^{T}=\left(\dot{A}^{T} \varepsilon\right) A$, we can see that

$$
\Omega=\left[\begin{array}{ccc}
0 & \Omega_{z} & -\Omega_{y} \\
-\Omega_{z} & 0 & \Omega_{x} \\
\Omega_{y} & -\Omega_{x} & 0
\end{array}\right],
$$

is an anti-symmetric matrix in generalized 3-space, i.e., $\Omega=-\Omega^{T} . \Omega$ is called the instantaneous rotation vector (Darboux vector) of the motion.

Theorem 1. The homothetic motions of generalized space $\mathrm{E}_{\alpha \beta}^{3}$ are regular motions.

Proof: Differentiating the equation $B=h A$ with respect to $t$ gives

$$
\dot{B}=\dot{h} A+\dot{A} h
$$

or

$$
\dot{B}=h\left(\frac{\dot{h}}{h} A+\dot{A}\right) .
$$

We may write $\operatorname{det} \dot{B}=h^{n} \cdot \operatorname{det}\left(\frac{\dot{h}}{h} A+\dot{A}\right)$. It is obviously, for any $t, \operatorname{det} \dot{B} \neq 0$.

\section{Pole Points and Pole Curves of the Motion}

To find the pole points, we have to solve the equation

$$
\dot{B} X+\dot{C}=0 .
$$

Any solution of the equation (3) is a pole point of the motion at that instant in $R_{\text {。 }}$. Since $\dot{B}$ is regular, the equation (3) has only one solution, i.e., $X_{\circ}=(-\dot{B})^{-1} \dot{C}=0$ at every instant $t$. This pole point in the fixed system is

$$
X=B(-\dot{B})^{-1} \dot{C}+C .
$$

Theorem 4. During the homothetic motion of generalized space of 3-dimensions, there is a unique instantaneous pole point at every time $t$.

\section{HAMILTON MOTIONS IN GENERALIZED 3-SAPCE}

Let us consider the curve $\gamma: I \subset \mathrm{R} \rightarrow \mathrm{E}_{\alpha \beta}^{4}$ defined by

$$
\gamma(t)=\left(a_{0}(t), a_{1}(t), a_{2}(t), a_{3}(t)\right)
$$

for every $t \in I$. We suppose that $\gamma(t)$ is a differentiable curve of order $r$ which does not pass through the origin.

Also, the map $F_{\gamma}$ acting on a pure quaternion $\omega$ is

$$
F_{\gamma}: \mathrm{K} \rightarrow \mathrm{K}, F_{\gamma}(\omega)=\gamma \circ \omega \circ \bar{\gamma},
$$

where $\bar{\gamma}$ is conjugate of the $\gamma$ and $\mathrm{K}$ is the set of all the pure generalized quaternions. We put $F_{\gamma}(\omega)=\omega^{\prime}$. Using the definition of $\stackrel{+}{H}$ and $\vec{H}$ the equation (5) is written as

From (1) and (2), we obtain

$$
\omega^{\prime}=\stackrel{+}{H}(\gamma) \stackrel{-}{H}(\bar{\gamma}) \omega .
$$




$$
\stackrel{+}{H}(\gamma) \bar{H}(\bar{\gamma})=\left[\begin{array}{cccc}
a_{0}^{2}+\alpha a_{1}^{2}+\beta a_{2}^{2}+\alpha \beta a_{3}^{2} & 0 & 0 & 0 \\
0 & a_{0}^{2}+\alpha a_{1}^{2}-\beta a_{2}^{2}-\alpha \beta a_{3}^{2} & 2 \beta\left(a_{1} a_{2}-a_{0} a_{3}\right) & 2 \beta\left(\alpha a_{1} a_{3}+a_{0} a_{2}\right) \\
0 & 2 \alpha\left(a_{1} a_{2}+a_{0} a_{3}\right) & a_{0}^{2}-\alpha a_{1}^{2}+\beta a_{2}^{2}-\alpha \beta a_{3}^{2} & 2 \alpha\left(\beta a_{2} a_{3}-a_{0} a_{1}\right) \\
0 & 2\left(\alpha a_{1} a_{3}-a_{0} a_{2}\right) & 2\left(a_{0} a_{1}+\beta a_{2} a_{3}\right) & a_{0}^{2}-\alpha a_{1}^{2}-\beta a_{2}^{2}+\alpha \beta a_{3}^{2}
\end{array}\right] .
$$

This simplifies to

$$
\stackrel{+}{H}(\gamma) \bar{H}(\bar{\gamma})=\left[\begin{array}{cc}
h^{\prime} & 0 \\
0 & B
\end{array}\right],
$$

where $h=N_{\gamma}=a_{0}^{2}+\alpha a_{1}^{2}+\beta a_{2}^{2}+\alpha \beta a_{3}^{2}$ and

$$
B=\left[b_{i j}\right]_{3 \times 3}=\left[\begin{array}{ccc}
a_{0}^{2}+\alpha a_{1}^{2}-\beta a_{2}^{2}-\alpha \beta a_{3}^{2} & 2 \beta\left(a_{1} a_{2}-a_{0} a_{3}\right) & 2 \beta\left(\alpha a_{1} a_{3}+a_{0} a_{2}\right) \\
2 \alpha\left(a_{1} a_{2}+a_{0} a_{3}\right) & a_{0}^{2}-\alpha a_{1}^{2}+\beta a_{2}^{2}-\alpha \beta a_{3}^{2} & 2 \alpha\left(\beta a_{2} a_{3}-a_{0} a_{1}\right) \\
2\left(\alpha a_{1} a_{3}-a_{0} a_{2}\right) & 2\left(a_{0} a_{1}+\beta a_{2} a_{3}\right) & a_{0}^{2}-\alpha a_{1}^{2}-\beta a_{2}^{2}+\alpha \beta a_{3}^{2}
\end{array}\right] .
$$

For the matrix $B$, we have $B^{T} \varepsilon B=h^{2} . \varepsilon$ and $\operatorname{det} B=h^{3}$.

The 1-parameter Hamilton motions of a body in generalized 3 - space are generated by transformation

$$
\left[\begin{array}{l}
X \\
1
\end{array}\right]=\left[\begin{array}{ll}
B & C \\
0 & 1
\end{array}\right]\left[\begin{array}{c}
X_{0} \\
1
\end{array}\right]
$$

where $B$ is the above matrix. $X, X_{0}$ and $C$ are $3 \times 1$ real matrices. $B$ and $C$ are continuously differentiable functions of a real parameter $t . X$ and $X_{0}$ correspond to the position vectors of the same point $P$.

Theorem 5. The Hamilton motion determined by the equation (6) is a homothetic motion in $\mathrm{E}_{\alpha \beta}^{3}$.

Proof: The matrix $B$ can be represented as

$$
B=h\left[\begin{array}{ccc}
\frac{b_{11}}{h} & \frac{b_{12}}{h} & \frac{b_{13}}{h} \\
\frac{b_{21}}{h} & \frac{b_{22}}{h} & \frac{b_{23}}{h} \\
\frac{b_{31}}{h} & \frac{b_{32}}{h} & \frac{b_{33}}{h}
\end{array}\right]=h A,
$$

where $h: I \subset \mathrm{R} \rightarrow \mathrm{R}$,

$$
t \rightarrow h(t)=a_{0}^{2}(t)+\alpha a_{1}^{2}(t)+\beta a_{2}^{2}(t)+\alpha \beta a_{3}^{2}(t) .
$$

So, we find $A \in Q O(3)$ and $h \in \mathrm{R}$.. Thus $B$ is a homothetic matrix and the equation (6) determines a homothetic motion.

\section{Special cases:}

1) If $\alpha=\beta=1$, then Theorem 5 holds for Euclidean three space $\mathrm{E}^{3}$ [5].

2) If $\alpha=1, \beta=-1$, then Theorem 5 holds for Minkowski three space $\mathrm{E}_{1}^{3}$ [9].

Example 1. Let $\gamma: I \subset \mathrm{R} \rightarrow \mathrm{E}_{\alpha \beta}^{4}$ be a curve given by

$$
t \rightarrow \gamma(t)=(\cos t, t, \sin t, 0),
$$

for every $t \in I . \gamma(t)$ is a differentiable regular of order $r$. Since, $\gamma(t)$ does not pass though the origin, the matrix $B$ can be represented as 
M. Jafari / Anadolu Univ. J.of Sci. and Tech. B-Theoretical Sci. 4 (1) - 2016

$$
\begin{aligned}
B & =\left[\begin{array}{ccc}
\cos ^{2} t+\alpha t^{2}-\beta \sin ^{2} t & 2 \beta t \sin t & 2 \beta(\cos t \sin t) \\
2 \alpha t \sin t & \cos ^{2} t-\alpha t^{2}+\beta \sin ^{2} t & 2 \alpha(-\mathrm{t} \cos t) \\
-2(\cos t \sin t) & 2(t \cos t) & \cos ^{2} t-\alpha t^{2}-\beta \sin ^{2} t
\end{array}\right] \\
& =\left(\cos ^{2} t+\alpha t^{2}+\beta \sin ^{2} t\right) A,
\end{aligned}
$$

where $h(t)=\cos ^{2} t+\alpha t^{2}+\beta \sin ^{2} t, A \in \mathrm{QO}(3)$. Thus, $B$ is a homothetic matrix and it determines a homothetic motion in $\mathrm{E}_{\alpha \beta}^{3}$.

\section{DARBOUX VECTOR OF THE MOTION}

In Euclidean 3-space, Yaylı [15] has showed the the Darboux vector of the homothetic motion which is defined by the Hamilton operators, can be written as multiplication of two real quaternions. In this section, we obtain the Darboux vector of the homothetic motion in generalized 3-space and show that it can be written as multiplication of two generalized quaternions.

Suppose that $\gamma(t)$ is a curve as defined in (4). The Darboux matrix in the homothetic motion defined by homothetic matrix $B$, is

So we obtain

$$
\Omega=B^{T} \varepsilon \dot{B}
$$

$$
\Omega=\frac{2}{h}\left[\begin{array}{ccc}
\frac{\dot{h}}{2} & \dot{a}_{3} a_{0}+\dot{a}_{2} a_{1}-\dot{a}_{1} a_{2}-\dot{a}_{0} a_{3} & -\left(\dot{a}_{2} a_{0}-\alpha \dot{a}_{3} a_{1}-\dot{a}_{0} a_{2}+\alpha \dot{a}_{1} a_{3}\right) \\
-\left(\dot{a}_{3} a_{0}+\dot{a}_{2} a_{1}-\dot{a}_{1} a_{2}-\dot{a}_{0} a_{3}\right) & \frac{\dot{h}}{2} & \dot{a}_{1} a_{0}-\dot{a}_{0} a_{1}+\beta \dot{a}_{3} a_{2}-\beta \dot{a}_{2} a_{3} \\
\dot{a}_{2} a_{0}-\alpha \dot{a}_{3} a_{1}-\dot{a}_{0} a_{2}+\alpha \dot{a}_{1} a_{3} & -\left(\dot{a}_{1} a_{0}-\dot{a}_{0} a_{1}+\beta \dot{a}_{3} a_{2}-\beta \dot{a}_{2} a_{3}\right) & \frac{\dot{h}}{2}
\end{array}\right] .
$$

We investigate the Darboux matrix in special case $h=1$. In the case, we have

$$
\Omega=\left[\begin{array}{ccc}
0 & \dot{a}_{3} a_{0}+\dot{a}_{2} a_{1}-\dot{a}_{1} a_{2}-\dot{a}_{0} a_{3} & -\left(\dot{a}_{2} a_{0}-\alpha \dot{a}_{3} a_{1}-\dot{a}_{0} a_{2}+\alpha \dot{a}_{1} a_{3}\right) \\
-\left(\dot{a}_{3} a_{0}+\dot{a}_{2} a_{1}-\dot{a}_{1} a_{2}-\dot{a}_{0} a_{3}\right) & 0 & \dot{a}_{1} a_{0}-\dot{a}_{0} a_{1}+\beta \dot{a}_{3} a_{2}-\beta \dot{a}_{2} a_{3} \\
\dot{a}_{2} a_{0}-\alpha \dot{a}_{3} a_{1}-\dot{a}_{0} a_{2}+\alpha \dot{a}_{1} a_{3} & -\left(\dot{a}_{1} a_{0}-\dot{a}_{0} a_{1}+\beta \dot{a}_{3} a_{2}-\beta \dot{a}_{2} a_{3}\right) & 0
\end{array}\right]
$$

The Darboux vector corresponds to skew-symmetric matrix $\Omega$ is defined by

$$
\vec{\Omega}=\left(\Omega_{x}, \Omega_{y}, \Omega_{z}\right)
$$

Therefore, the Darboux vector of the motion

$$
\vec{\Omega}=2\left(\dot{a}_{1} a_{0}-\dot{a}_{0} a_{1}+\beta \dot{a}_{3} a_{2}-\beta \dot{a}_{2} a_{3}, \dot{a}_{2} a_{0}-\alpha \dot{a}_{3} a_{1}-\dot{a}_{0} a_{2}+\alpha \dot{a}_{1} a_{3}, \dot{a}_{3} a_{0}+\dot{a}_{2} a_{1}-\dot{a}_{1} a_{2}-\dot{a}_{0} a_{3}\right),
$$

is obtained. This vector can be written as multiplication of two generalized quaternions as

$$
\vec{\Omega}=2(\dot{\gamma} \circ \bar{\gamma})
$$

\section{Special cases:}

1. $\alpha=\beta=1$, is considered, then $\vec{\Omega}$ is Darboux vector for homothetic motion in $\mathrm{E}^{3}$ [5].

2. $\alpha=1, \beta=-1$, is considered, then $\vec{\Omega}$ is Darboux vector for homothetic motion in $\mathrm{E}_{1}^{3}[9]$. 
Example 2. Let $\gamma: I \subset \mathrm{R} \rightarrow \mathrm{E}_{\alpha \beta}^{4}$ be a curve given by

$$
\gamma(t)=\frac{1}{2}\left(\cos t, \frac{1}{\sqrt{\alpha}} \sin t, \sqrt{\frac{3}{\beta}}, 0\right),
$$

for every $t \in I . \gamma(t)$ is a differentiable regular of order $r$. Since, $\gamma(t)$ does not pass though the origin, the matrix $B$ can be represented as

$$
B=\left[\begin{array}{ccc}
-\frac{1}{2} & \frac{\sqrt{3 \beta}}{2 \sqrt{\alpha}} \sin t & \frac{\sqrt{3 \beta}}{2} \cos t \\
\frac{\sqrt{3 \alpha}}{2 \sqrt{\beta}} \sin t & \frac{1}{4}\left(\cos ^{2} t-\sin ^{2} t\right)+\frac{3}{4} & -\frac{\sqrt{\alpha}}{2} \sin t \cos t \\
-\frac{\sqrt{3}}{2 \sqrt{\beta}} \cos t & \frac{1}{2 \sqrt{\alpha}} \sin t \cos t & \frac{1}{4}\left(\cos ^{2} t-\sin ^{2} t\right)-\frac{3}{4}
\end{array}\right] .
$$

$B$ is a homothetic matrix and is defined a homothetic motion. The Darboux vector of this motion is

$$
\vec{\Omega}=\left(\frac{1}{2 \sqrt{\alpha}}(\sin t+\cos t), \frac{\sqrt{3}}{4 \sqrt{\beta}} \sin t,-\frac{\sqrt{3}}{4 \sqrt{\alpha \beta}} \cos t\right) .
$$

\section{REFERENCES}

[1] Bottema O, Roth B, Theorical kinematics, Oxford, New York; 1979.

[2] Hacisalihoglu H.H, On the rolling of one curve or surface upon another, Mathematical proceedings of the Royal Irish academy, 71A2; 13-17, 1971

[3] Jafari, M. On the properties of quasi-quaternion algebra, Communications faculty of science, university of Ankara, Series1, 63(1); 1-10, 2014.

[4] Jafari M, Yayli Y, Rotation in four dimensions via generalized Hamilton operators, Kuwait journal of science, 40(1); 67-79, 2013.

[5] Jafari M, Homothetic motions in Euclidean 3-space, Kuwait journal of science, 41(1); 65-73, 2014.

[6] Jafari $\mathrm{M}$, Yayli $\mathrm{Y}$, Homothetic Motions at $\mathrm{E}_{\alpha \beta}^{4}$, International Journal contemporary of Mathematical Sciences, 5 (47); 2319-2326, 2010.

[7] Jafari M, Yayli Y, Generalized quaternions and rotation in 3-space $E_{\alpha \beta}^{3}$, TWMS journal of pure and applied mathematics, 6(2); 224-235, 2015.

[8] Jafari M, Split semi-quaternions algebra in semi-Euclidean 4-space, Cumhuriyet university faculty of science, 36 (1); 70-77, 2015.

[9] Jafari M, Yayli Y, One-parameter homothetic motions in the Minkowski 3-space, TWMS Journal of Applied and Engineering Mathematics, 6(1); 335-346, 2016. 
[10] Kula L, Yayli Y, Split quaternions and rotations in semi-Euclidean space $E_{2}^{4}$, Journal of Korean mathematical society, 44(6); 1313-1327, 2007.

[11] Mortazaasl H, Jafari M, A study on semi-quaternions algebra in semi-Euclidean 4-space, mathematical science and application E-Notes, 1(2); 20-27, 2013.

[12] Müller H.R, Kinematik dersleri, Ankara üniversitesi fen fakultesi yaynları, Um. 96 (Mat No 2, 1963.

[13] Tosun M, Kucuk A, Gungor M.A, The homothetic motions in the Lorentz 3-space, Acta mathematica science, 26B(4); 711-719. 2006

[14] Yaylı Y, Homothetic motions at E ${ }^{4}$, Mechanism and machine theory, 27(3); 303-305, 1992.

[15] Yaylı Y, Hamilton operators and Lie groups, Ph.D. thesis, Gazi University, Ankara, Turkey, 1988. 